\title{
Glucosuria Is Not Always Due to Diabetes
}

Capt Meghan Lewis, MD, USAF, MC; and Bhagwan Dass, MD

\author{
Further study of the long-term implications and follow-up is needed on SGLT2 mutation, an \\ uncommon cause of glucosuria that mimics the effect of SGLT2 inhibitors, including the possi- \\ ble development of further renal disease, type 2 diabetes mellitus, and cardiovascular disease.
}

\begin{abstract}
Meghan Lewis is a Resident Physician, and Bhagwan Dass is a Staff Physician, both at Eglin Air Force Base in Florida. Bhagwan Dass is an Associate Professor at the University of Florida in Gainesville. Correspondence: Bhagwan Dass (bdass98@gmail.com)
\end{abstract}

Fed Pract. 2021;38(2):92-95. doi:10.12788/fp.0091
5 amilial renal glucosuria is an uncommon, rarely documented condition wherein the absence of other renal or endocrine conditions and with a normal serum glucose level, glucosuria persists due to an isolated defect in the nephron's proximal tubule. Seemingly, in these patients, the body's physiologic function mimics that of sodiumglucose cotransporter-2 (SGLT2)-inhibiting medications with the glucose cotransporter being selectively targeted for promoting renal excretion of glucose. This has implications for the patient's prospective development of hyperglycemic diseases, urinary tract infections (UTIs), and potentially even cardiovascular disease. Though it is a generally asymptomatic condition, it is one that seasoned clinicians should investigate given the future impacts and considerations required for their patients.

\section{CASE PRESENTATION}

Mr. A was a 28-year-old male with no medical history nor prescription medication use who presented to the nephrology clinic at Eglin Air Force Base, Florida, in June 2019 for a workup of asymptomatic glucosuria. The condition was discovered on a routine urinalysis in October 2015 at the initial presentation at Eglin Air Force Base, when the patient was being evaluated by his primary care physician for acute, benign headache with fever and chills. Urinalysis testing was performed in October 2015 and resulted in a urine glucose of $500 \mathrm{mg} / \mathrm{dL}(2+)$. He was directed to the emergency department for further evaluation, reciprocating the results.

On further laboratory testing in October 2015, his blood glucose was normal at $75 \mathrm{mg} / \mathrm{dL}$; hemoglobin $A_{1 c}$ was $5.5 \%$. On repeat urinalysis 2 weeks later, his urinary glucose was found to be $500 \mathrm{mg} / \mathrm{dL}(2+)$. Each time, the elevated urinary glucose was the only abnor- mal finding: There was no concurrent hematuria, proteinuria, or ketonuria. The patient reported he had no associated symptoms, including nausea, vomiting, abdominal pain, dysuria, polyuria, and increased thirst. He was not taking any prescription medications, including SGLT2 inhibitors. His presenting headache and fever resolved with supportive care and was considered unrelated to his additional workup.

A diagnostic evaluation ensued from 2015 to 2020, including follow-up urinalyses, metabolic panels, complete blood counts, urine protein electrophoresis (UPEP), urine creatinine, urine electrolytes, $25-\mathrm{OH}$ vitamin D level, $\kappa / \lambda$ light chain panel, and serum protein electrophoresis (SPEP). The results of all diagnostic workup throughout the entirety of his evaluation were found to be normal. In 2020 , his $25-\mathrm{OH}$ vitamin D level was borderline low at $29.4 \mathrm{ng} / \mathrm{mL}$. His $\kappa / \lambda$ ratio was normal at 1.65 , and his serum albumin protein electrophoresis was $4.74 \mathrm{~g} / \mathrm{dL}$, marginally elevated, but his SPEP and UPEP were normal, as were urine protein levels, total gamma globulin, and no monoclonal gamma spike noted on pathology review. Serum uric acid, and urine phosphorous were both normal. His serum creatinine and electrolytes were all within normal limits. Over the 5 years of intermittent monitoring, the maximum amount of glucosuria was $1,000 \mathrm{mg} / \mathrm{dL}$ (3+) and the minimum was $250 \mathrm{mg} / \mathrm{dL}(1+)$. There was a gap of monitoring from March 2016 until June 2019 due to the patient receiving care from offsite health care providers without shared documentation of specific laboratory values, but notes documenting persistent glucosuria (Table).

\section{Analysis}

Building the initial differential diagnosis for this patient began with confirming that 
he had isolated glucosuria, and not glucosuria secondary to elevated serum glucose. Additionally, conditions related to generalized proximal tubule dysfunction, acute or chronic impaired renal function, and neoplasms, including multiple myeloma (MM), were eliminated because this patient did not have the other specific findings associated with these conditions.

Proximal tubulopathies, including proximal renal tubular acidosis (type 2) and Fanconi syndrome, was initially a leading diagnosis in this patient. Isolated proximal renal tubular acidosis (RTA) (type 2) is uncommon and pathophysiologically involves reduced proximal tubular reabsorption of bicarbonate, resulting in low serum bicarbonate and metabolic acidosis. Patients with isolated proximal RTA (type 2) typically present in infancy with failure to thrive, tachypnea, recurrent vomiting, and feeding difficulties. These symptoms do not meet our patient's clinical presentation. Fanconi syndrome involves a specific disruption in the proximal tubular apical sodium uptake mechanism affecting the transmembrane sodium gradient and the sodium-potassiumATPase pump. Fanconi syndrome, therefore, would not only present with glucosuria, but also classically with proteinuria, hypophosphatemia, hypokalemia, and a hyperchloremic metabolic acidosis.

Chronic or acute renal disease may present with glucosuria, but one would expect additional findings including elevated serum creatinine, elevated urinary creatinine, 25-OH vitamin D deficiency, or anemia of chronic disease. Other potential diagnoses included MM and similar neoplasms. MM also would present with glucosuria with proteinuria, an elevated $\kappa / \lambda$ light chain ratio, and an elevated SPEP and concern for bone lytic lesions, which were not present. A related disorder, monoclonal gammopathy of renal significance (MGRS), akin to monoclonal gammopathy of unknown significance (MGUS), presents with proteinuria with evidence of renal injury. While this patient had a marginally elevated $\kappa / \lambda$ light chain ratio, the remainder of his SPEP and UPEP were normal, and evaluation by a hematologist/ oncologist and pathology review of laboratory findings confirmed no additional evidence for MM, including no monoclonal $\gamma$
TABLE Patient's Urine and Serum Glucose Levels, 2015-2020

\begin{tabular}{lccc} 
Dates & $\begin{array}{c}\text { Urine Glucose, } \\
\mathbf{m g} / \mathbf{d L}\end{array}$ & $\begin{array}{c}\text { Urine-Specific } \\
\text { Gravity }\end{array}$ & $\begin{array}{c}\text { Serum Glucose, } \\
\mathbf{m g} / \mathbf{d L}\end{array}$ \\
\hline October 2015 & $500(2+)$ & 1.020 & 75 \\
\hline November 2015 & $500(2+)$ & 1.025 & Not performed \\
\hline March 2016 & $250(1+)$ & 1.025 & 86 \\
\hline June 2019 & $250(1+)$ & 1.013 & 88 \\
\hline July 2019 & $250(1+)$ & 1.025 & 95 \\
\hline September 2019 & $1,000(3+)$ & 1.020 & 90 \\
\hline October 2019 & $250(1+)$ & 1.004 & 78 \\
\hline March 2020 & $250(1+)$ & 1.021 & 97
\end{tabular}

spike. With no evidence of renal injury with a normal serum creatinine and glomerular filtration rate, MGRS was eliminated from the differential as it did not meet the International Myeloma Working Group diagnostic criteria. ${ }^{1}$ The elevated $\kappa / \lambda$ ratio with normal renal function is attributed to polyclonal immunoglobulin elevation, which may occur more commonly with uncomplicated acute viral illnesses.

\section{Diagnosis}

The differential homed in on a targeted defect in the proximal tubular SGLT2 gene as the final diagnosis causing isolated glucosuria. Familial renal glucosuria (FRG), a condition caused by a mutation in the SLC5A2 gene that codes for the SGLT2 has been identified in the literature as causing cases with nearly identical presentations to this patient. ${ }^{2,3}$ This condition is often found in otherwise healthy, asymptomatic patients in whom isolated glucosuria was identified on routine urinalysis testing.

Due to isolated case reports sharing this finding and the asymptomatic nature of the condition, specific data pertaining to its prevalence are not available. Case studies of other affected individuals have not noted adverse effects (AEs), such as UTIs or hypotension specifically. ${ }^{2,3}$ The patient was referred for genetic testing for this gene mutation; however, he was unable to obtain the test due to lack of insurance coverage. Mr. A has no other family members that have been evaluated for 
FIGURE Sodium-Glucose Cotransporter in the Renal Proximal Convoluted Tubule

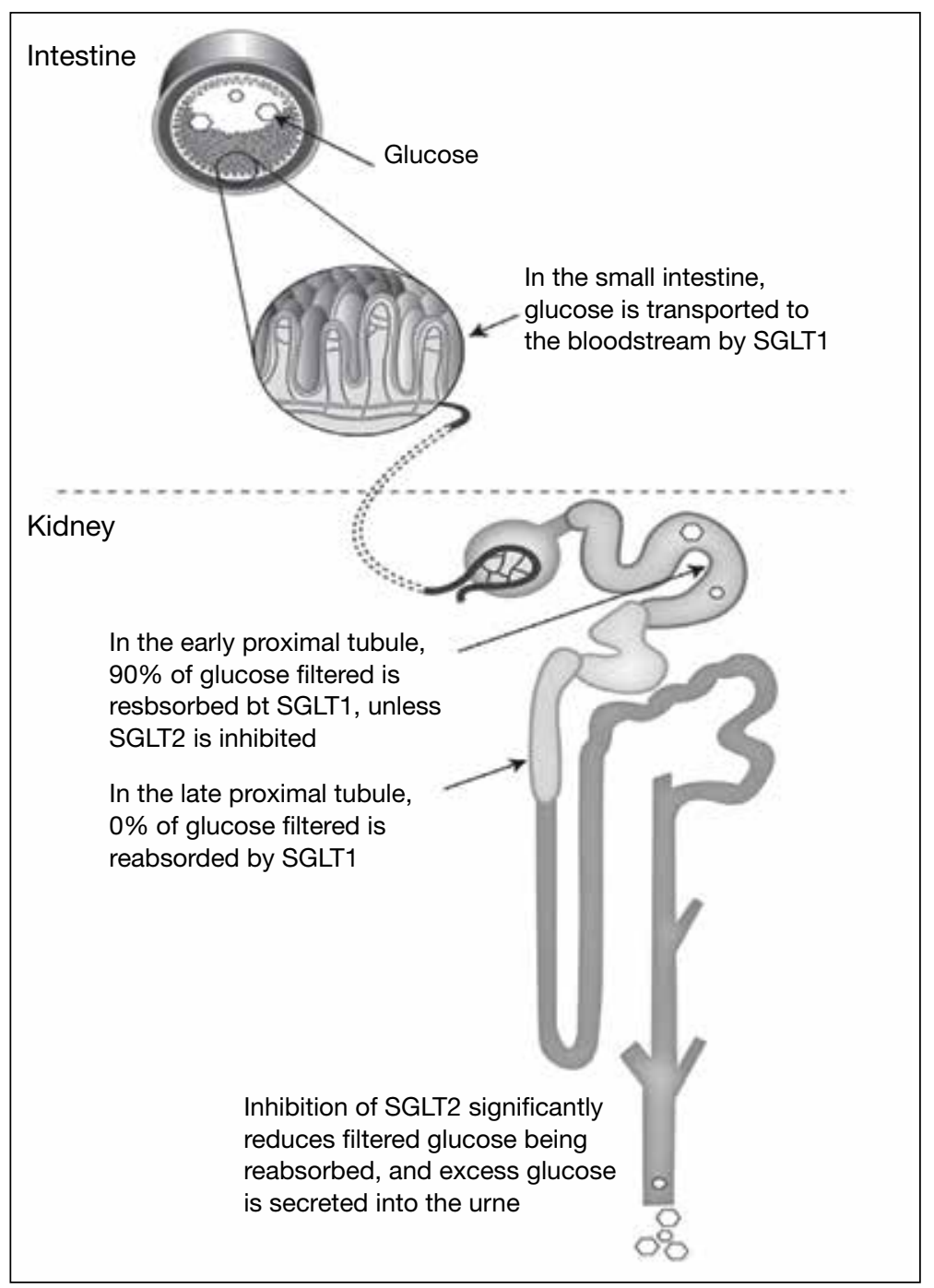

Reprinted with permission. Neumiller JJ. Empagliflozin: a new sodium-glucose cotransporter 2 (SGLT2) inhibitor for the treatment of type 2 diabetes. Drugs Context. 2014;3:212262. Published 2014 Jun 11. doi:10.7573/dic.212262

or identified as having this condition. Despite the name, FRG has an unknown inheritance pattern and is attributed to a variety of missense mutations in the SLC5A2 gene.,

\section{DISCUSSION}

The SGLT2 gene believed to be mutated in this patient has recently become wellknown. The inhibition of the SGLT2 transport protein has become an important tool in the management of type 2 diabetes mellitus (T2DM) independent of the insulin pathway. The SGLT2 in the proximal convoluted tubule of the kidney reabsorbs the majority,
$98 \%$, of the renal glucose for reabsorption, and the remaining glucose is reabsorbed by the SGLT2 gene in the more distal portion of the proximal tubule in healthy individuals. ${ }^{4,6}$ The normal renal threshold for glucose reabsorption in a patient with a normal glomerular filtration rate is equivalent to a serum glucose concentration of $180 \mathrm{mg} / \mathrm{dL}$, even higher in patients with T2DM due to upregulation of the SGLT2 inhibitors. SGLT2 inhibitors, such as canagliflozin, dapagliflozin, and empagliflozin, selectively inhibit this cotransporter, reducing the threshold from 40 to $120 \mathrm{mg} / \mathrm{dL}$, thereby significantly increasing the renal excretion of glucose. ${ }^{4}$ The patient's mutation in question and clinical presentation aligned with a naturally occurring mimicry of this drug's mechanism of action (Figure).

Arguably, one of the more significant benefits to using this new class of oral antihyperglycemics, aside from the noninferior glycemic control compared with that of other first-line agents, is the added metabolic benefit. To date, SGLT2 inhibitors have been found to decrease blood pressure in all studies of the medications and promote moderate weight loss. ${ }^{7}$ SGLT2 inhibitors have not only demonstrated significant cardiovascular (CV) benefits, linked with the aforementioned metabolic benefits, but also have reduced hospitalizations for heart failure in patients with T2DM and those without. ${ }^{7}$ The EMPA-REG OUTCOME trial showed a 38\% relative risk reduction in $\mathrm{CV}$ events in empagliflozin vs placebo. ${ }^{4,8}$ However, it is unknown whether patients with the SLC5A2 mutation also benefit from these $\mathrm{CV}$ benefits akin to the SGLT2 inhibiting medications, and it is and worthy of studying via longterm follow-up with patients similar to this.

This SLC5A2 mutation causing FRG selectively inhibiting SGLT2 function effectively causes this patient's natural physiology to mimic that of these new oral antihyperglycemic medications. Patients with FRG should be counseled regarding this condition and the implications it has on their overall health. At this time, there is no formal recommendation for short-term or longterm management of patients with FRG; observation and routine preventive care monitoring based on US Preventive Services Task Force screening recommendations 
apply to this population in line with the general population.

This condition is not known to be associated with hypotension or hypoglycemia, and to some extent, it can be theorized that patients with this condition may have inherent protection of development of hyperglycemia. ${ }^{4}$ Akin to patients on SGLT2 inhibitors, these patients may be at an increased risk of UTIs and genital infections, including mycotic infections due to glycemic-related imbalance in the normal flora of the urinary tract. ${ }^{9}$ Other serious AEs of SGLT2 inhibitors, such as diabetic ketoacidosis, osteoporosis and related fractures, and acute pancreatitis, should be shared with FRG patients, though they are unlikely to be at increased risk for this condition in the setting of normal serum glucose and electrolyte levels. Notably, the osteoporosis risk is small, and specific other risk factors pertinent to individual patient's medical history, and canagliflozin exclusively. If a patient with FRG develops T2DM after diagnosis, it is imperative that they inform physicians of their condition, because SGLT2-inhibiting drugs will be ineffective in this subset of patients, necessitating increased clinical judgment in selecting an appropriate antihyperglycemic agent in this population.

\section{CONCLUSIONS}

FRG is an uncommon diagnosis of exclusion that presents with isolated glucosuria in the setting of normal serum glucose. The patient generally presents asymptomatically with a urinalysis completed for other reasons, and the patient may or may not have a family history of similar findings. The condition is of particular interest given that its SGLT2 mutation mimics the effect of SGLT2 inhibitors used for T2DM. More monitoring of patients with this condition will be required for documentation regarding long-term implications, including development of further renal disease, $\mathrm{T} 2 \mathrm{DM}$, or CV disease.

\section{Author disclosures}

The authors report no actual or potential conflicts of interest with regard to this article.

\section{Disclaimer}

The opinions expressed herein are those of the authors and do not necessarily reflect those of Federal Practitioner, Frontline Medical Communications Inc., the US Government, or any of its agencies.

\section{References}

1. Rajkumar SV, Dimopoulos MA, Palumbo A, et al. International Myeloma Working Group updated criteria for the diagnosis of multiple myeloma. Lancet Oncol. 2014;15(12). doi:10.1016/s1470-2045(14)70442-5

2. Calado J, Sznajer Y, Metzger D, et al. Twenty-one additional cases of familial renal glucosuria: absence of genetic heterogeneity, high prevalence of private mutations and further evidence of volume depletion. Nephrol Dial Transplant. 2008;23(12):3874-3879. doi.org/10.1093/ndt/gfn386

3. Kim KM, Kwon SK, Kim HY. A case of isolated glycosuria mediated by an SLC5A2 gene mutation and characterized by postprandial heavy glycosuria without salt wasting. Electrolyte Blood Press. 2016;14(2):35-37. doi:10.5049/EBP.2016.14.2.35

4. Hsia DS, Grove O, Cefalu WT. An update on sodiumglucose co-transporter-2 inhibitors for the treatment of diabetes mellitus. Curr Opin Endocrinol Diabetes Obes. 2017;24(1):73-79. doi:10.1097/MED.0000000000000311

5. Kleta R. Renal glucosuria due to SGLT2 mutations. Mol Genet Metab. 2004;82(1):56-58. doi:10.1016/j.ymgme.2004.01.018

6. Neumiller JJ. Empagliflozin: a new sodium-glucose co-transporter 2 (SGLT2) inhibitor for the treatment of type 2 diabetes. Drugs Context. 2014;3:212262. doi:10.7573/dic.212262

7. Raz I, Cernea S, Cahn A. SGLT2 inhibitors for primary prevention of cardiovascular events. J Diabetes. 2020;12(1):57. doi:10.1111/1753-0407.13004

8. Zinman B, Wanner C, Lachin JM, et al. Empagliflozin, cardiovascular outcomes, and mortality in type 2 diabetes. N Engl J Med. 2015;373(22):2117-2128. doi:10.1056/nejmoa1504720

9. Mcgill JB, Subramanian S. Safety of sodium-glucose cotransporter 2 inhibitors. Am J Cardiol. 2019;124(suppl 1):S45-S52. doi:10.1016/j.amjcard.2019.10.029 